\title{
Markers of Inflammation Associated with Plaque Progression and Instability in Patients with Carotid Atherosclerosis
}

\author{
Enrico Ammirati, ${ }^{1,2}$ Francesco Moroni, ${ }^{1}$ Giuseppe Danilo Norata, ${ }^{3,4,5}$ \\ Marco Magnoni, ${ }^{1}$ and Paolo G. Camici ${ }^{1}$ \\ ${ }^{1}$ Cardiothoracic Department, San Raffaele Scientific Institute and Vita-Salute San Raffaele University, \\ Via Olgettina 58-60, 20132 Milan, Italy \\ ${ }^{2}$ Cardiovascular and Thoracic Department, Niguarda Ca' Granda Hospital, Milan, Italy \\ ${ }^{3}$ Departement of Pharmacological and Biomolecular Sciences, Università degli Studi di Milano, Milan, Italy \\ ${ }^{4}$ Center for the Study of Atherosclerosis, Bassini Hospital, Cinisello Balsamo, Italy \\ ${ }^{5}$ Blizard Institute, Queen Mary University, London, UK
}

Correspondence should be addressed to Enrico Ammirati; ammirati.enrico@hsr.it

Received 23 February 2015; Accepted 22 March 2015

Academic Editor: Marc Pouliot

Copyright ( 2015 Enrico Ammirati et al. This is an open access article distributed under the Creative Commons Attribution License, which permits unrestricted use, distribution, and reproduction in any medium, provided the original work is properly cited.

\begin{abstract}
Atherosclerosis is the focal expression of a systemic disease affecting medium- and large-sized arteries, in which traditional cardiovascular risk factor and immune factors play a key role. It is well accepted that circulating biomarkers, including C-reactive protein and interleukin-6, reliably predict major cardiovascular events, including myocardial infarction or death. However, the relevance of biomarkers of systemic inflammation to atherosclerosis progression in the carotid artery is less established. The large majority of clinical studies focused on the association between biomarkers and subclinical atherosclerosis, that is, carotid intimamedia thickening (cIMT), which represents an earlier stage of the disease. The aim of this work is to review inflammatory biomarkers that were associated with a higher atherosclerotic burden, a faster disease progression, and features of plaque instability, such as inflammation or neovascularization, in patients with carotid atherosclerotic plaque, which represents an advanced stage of disease compared with cIMT. The association of biomarkers with the occurrence of cerebrovascular events, secondary to carotid plaque rupture, will also be presented. Currently, the degree of carotid artery stenosis is used to predict the risk of future cerebrovascular events in patients affected by carotid atherosclerosis. However, this strategy appears suboptimal. The identification of suitable biomarkers could provide a useful adjunctive criterion to ensure better risk stratification and optimize management.
\end{abstract}

\section{Introduction}

Atherosclerotic disease of the carotid arteries appears to be highly prevalent in the ageing population. According to recent epidemiological surveys, up to $5 \%$ of women and $12 \%$ of men over the age of 80 suffer from asymptomatic moderate (i.e., reduction in diameter between $50 \%$ and $70 \%$ ) atherosclerotic carotid artery stenosis, while $1 \%$ and $3 \%$, respectively, suffer from severe carotid stenosis, defined as a reduction in diameter of $\geq 70 \%$ [1]. It is well recognized that the presence of atherosclerotic disease in the carotid arteries poses a substantial risk of ipsilateral cerebrovascular events, with reported annual ischemic stroke rates ranging from $0.35 \%$ to $1.3 \%$ in asymptomatic patients with moderate stenosis $[2,3]$ and from $0.5 \%$ to approximately $5 \%$ for severe asymptomatic carotid artery stenosis $[2,4]$. Around $20 \%$ of ischemic strokes appear to originate from carotid plaques [5], mainly due to an arterioarterial embolization [6], calling for the institution of appropriate management strategies aimed at effectively minimizing risk. Current guidelines recommend surgical or endovascular revascularization in patients bearing an asymptomatic carotid artery stenosis of $\geq 60 \%$ and with a life expectancy of more than 5 years [7]. The appropriateness of such indication is, however, challenged by the evidence provided by randomized controlled trials and meta-analyses that have shown no benefit of revascularization over optimal 
medical therapy in the management of asymptomatic carotid stenosis $[8,9]$. Current risk stratification strategies appear to be inadequate in reliably identifying individuals who may benefit from aggressive treatment $[10,11]$.

Apart from the risk of stroke, the presence of severe asymptomatic carotid artery stenosis was reproducibly shown to be associated with cognitive decline $[12,13]$. The pathogenic mechanism appears to involve both chronic hypoperfusion [14] and microembolic events [15]. The possibility of developing mild cognitive impairment may thus be taken into account when choosing the appropriate management strategy for asymptomatic carotid artery stenosis.

Several serum inflammatory markers have been proposed as tools for risk assessment in patients bearing atherosclerotic lesions of the carotid artery. Among them, notable examples include fibrinogen, serum amyloid A (SAA), interleukin6 (IL-6), and lipoprotein-associated phospholipase A2 (LpPLA2), which was recently approved by United States Food and Drugs Administration as a predictor of ischemic stroke $[16,17]$. The most widely used in current clinical practice however remains high sensitivity C-reactive protein (hsCRP), which was shown to reproducibly predict the risk of stroke in several large epidemiological studies, including the Physicians' Health Study [18], Women's Health Study [19], and the Framingham Heart Study [20]. In addition, the in vivo identification of plaque features predictive of progression or instability would be advantageous in establishing the appropriate clinical management, including imaging follow-up and therapeutic interventions. Serum biomarkers reflecting the activity of biological processes involved in plaque growth or destabilization may provide great help for this purpose.

Of note, early atherosclerosis, measured as carotid intima-media thickness (cIMT), correlates with the risk of cardiovascular events in the general population. However, an association between changes in CIMT and the risk of cardiovascular events (including myocardial infarction, stroke, vascular death, or a combination of these) remained unproven in a large survey of more than 36000 subjects from the general population [21]. Despite this, patients with the highest "inflammatory load" had the greater cIMT progression [22], further underlining the relevance of serum inflammatory biomarkers as surrogates that could reflect processes associated with atherosclerotic disease progression. In the present work we critically review the major studies identifying inflammatory biomarkers associated with atherosclerotic disease progression, features of plaque vulnerability (including inflammation and neovascularization), or cerebrovascular symptoms, expanding and updating the concepts expressed by Hermus et al. in a previous article [23]. Table 1 summarizes the current available evidence on the association between carotid atherosclerosis and biomarkers.

Search Strategy. We searched PubMed electronic database, limiting the search to original research published between 1990 and December 2014. The key words used for the search were carotid stenosis and markers of inflammation, carotid stenosis and $T$ cells, carotid stenosis and monocytes, carotid plaque neovascularization and markers of inflammation, carotid plaque vulnerability and markers of inflammation.

\section{Inflammatory Biomarkers and Carotid Artery Plaque}

2.1. Markers of Inflammation and Degree of Stenosis. Identifying patients at risk of bearing an asymptomatic carotid artery stenosis appears of great importance for the implementation of reliable yet cost-effective ultrasonographic screening programs. Serum markers of inflammation may appropriately serve this purpose. As mentioned earlier, CRP is commonly used for cardiovascular risk evaluation. Recent reports have shown that higher serum hs-CRP levels are in fact able to predict the presence of carotid artery plaque [24, 25] but do not associate with the degree of stenosis the lesions determine [24]. These data contrast with previous reports on larger populations, which failed to demonstrate a similar association. In particular, Halvorsen and colleagues could not establish an association between CRP and presence of carotid plaque in a cross-sectional study involving 5341 individuals, both males and females [26], and similarly Chapman et al. obtained a similar result in a cohort of 1111 subjects [27].

The association between the presence and severity of carotid stenosis and other acute phase proteins of the pentraxin superfamily, which includes CRP, such as the long pentraxin-3 (PTX3), is not clear $[28,29]$. While PTX3 was shown to be atheroprotective in experimental atherosclerosis $[30,31]$, preliminary data in humans suggest that plasma levels of PTX3 are associated with the presence of atherosclerotic plaques and prevalent vascular disease [32]. A subsequent population-based study involving more than 2400 subjects, however, showed that PTX3 is not a predictor of incident cardiovascular events [33].

Other markers have been explored, including fibrinogen, which was more reliably associated with the presence of plaque than CRP [24, 26, 27], sVCAM [25], and erythrocyte sedimentation rate (ESR) [24], all of which showed some association with carotid atherosclerosis. Of great interest was the fact that IL-6, a master proinflammatory cytokine, was reproducibly shown to be higher in the serum of subjects bearing an asymptomatic carotid stenosis with respect to controls $[24,25,27]$. S100A12, a calcium binding protein involved in inflammatory signaling, was also shown to be elevated in patients bearing carotid atherosclerotic lesions [34]. Notably, none of these markers was shown to correlate with the degree of stenosis. On the other hand, serum levels of TNF $\alpha$ and L-selectin, a lectin type adhesion molecule expressed on leukocytes surface, were shown to associate with larger plaque size estimated by ultrasound imaging in a cohort of 1016 subjects [35].

2.2. Markers of Inflammation and Plaque Instability. Plaques at higher risk of causing an acute atherothrombotic or atheroembolic event, including stroke and acute myocardial infarction, that is, unstable plaques, share some distinctive features, such as a thinner fibrous cap overlying a large necrotic core or a strong intraplaque inflammatory reaction $[36,37]$. The detection of potentially unstable atherosclerotic lesions using biomarkers would bring obvious advantages in establishing an appropriate treatment plan in patients with an asymptomatic carotid stenosis. Features of plaque instability 


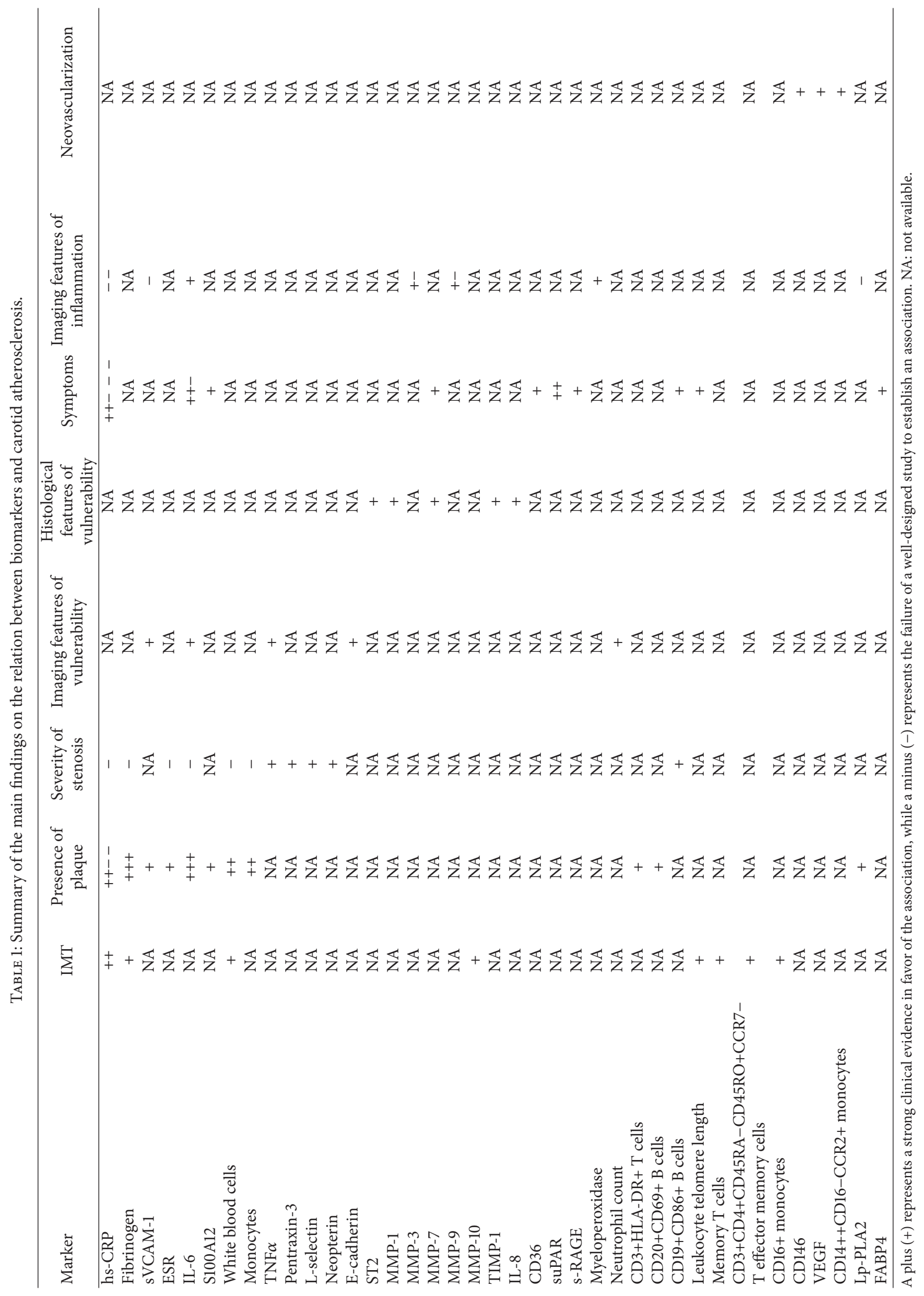


as evaluated by magnetic resonance imaging (MRI), that is, signal hypointensity in T1 weighted images, were shown to correlate with the upregulation of several proinflammatory molecules, such as the cytokines IL- 6 and TNF $\alpha$, the endothelial activation markers E-cadherin and VCAM-1, and the inflammatory markers hs-CRP and, most notably, PTX3 [38]. A recent report by Willems et al. failed to show an association between serum concentration of ST2, a member of IL1 receptor family, and a vulnerable plaque phenotype, as determined histologically [39]. Sugioka and colleagues have recently shown that serum levels of neopterin, a product of the catabolism of guanosine triphosphate secreted by macrophages upon activation, correlated with the presence of highly complex carotid lesions, suggestive of plaque vulnerability, in patients affected by stable coronary artery disease [40]. In a cohort of 101 patients, Pelisek and colleagues were able to establish an association between histological features of carotid plaque instability and serum levels of circulating matrix metalloproteinase (MMP)-1, MMP-7, tissue inhibitor of matrix protease (TIMP)-1, and IL-8 [41]. MMPs are a class of proteases involved in extracellular matrix degradation, which appear to play a key role in the process of vascular remodeling during the course of vascular disease [42]. The fact that numerous experimental studies suggest that MMPs may be involved in the process of plaque destabilization $[43,44]$ makes this finding particularly intriguing.

\subsection{Markers of Inflammation and Neurological Symptoms.} The ultimate goal of managing a patient suffering from asymptomatic carotid artery stenosis is, as reiterated above, preventing the occurrence of atheroembolic events leading to ischemic stroke. Studying patients soon after a cerebrovascular event of carotid origin might lead to the identification of serum markers potentially useful in identifying patients at higher risk for recurrence. Clearly, results obtained in the acute phase of disease should, however, be considered critically, since the acute process within the central nervous system may directly influence the concentration of circulating biomarkers, increasing the risk of spurious associations. Again, the analysis of hs-CRP has led to contradictory results, making it rather unreliable for the identification of patients deserving an aggressive management $[24,25$, $45,46]$. IL-6, on the other hand, was shown to be more reproducibly associated with the presence of a symptomatic carotid stenosis, but the results are far from being conclusive [24, 25, 45]. Among other inflammatory markers explored, the soluble form of CD36, a macrophage scavenger receptor involved in LDL uptake, was shown to correlate with the presence of symptoms and ultrasound features of plaque vulnerability in a cohort of 62 patients, 31 of which had suffered from stroke in the previous 6 months [47]. Interestingly, asymptomatic and symptomatic patients did not differ in terms of CRP, glycosylated hemoglobin, or lipid profile [47]. Plasma soluble urokinase plasminogen activator receptor (suPAR) is the soluble form of the cellsurface urokinase plasminogen activator receptor, released by endothelial and immune cells by proteolytic cleavage in an inflammatory environment [48]. Plasma levels of suPAR were higher in symptomatic patients than in patients bearing an asymptomatic carotid artery stenosis in a recent report on 162 patients [49]. In addition, suPAR was higher in patients suffering from stroke or TIA than symptomatic patients in which carotid atherosclerosis had manifested as amaurosis fugax [50]. Another soluble form of a receptor, the receptor for advanced glycosylation end products (RAGE), which is involved in pattern recognition, was shown to associate with the presence of symptoms in patients affected by carotid artery atherosclerosis, albeit in a small study comprising only 29 patients [51]. Consistent with its association with plaque features of instability, MMP-7 was shown to be elevated in the sera of patients who had a stroke from 2 to 6 months prior to the analysis [52].

\section{Systemic versus Local Inflammation}

Inflammation of blood vessel wall is a critical component of atherosclerosis and brings about several pathological changes such as edema, vasa vasorum dilation and proliferation, and immune cells infiltration [53]. The knowledge of the biological basis of these processes has led to the development of novel noninvasive imaging technique able to disclose them in vivo through the use of appropriately designed molecular probes $[36,54]$. In this respect, positron emission tomography (PET) using the radiolabelled glucose analogue $18 \mathrm{~F}$ fluorodeoxyglucose (FDG) is the most widely used imaging tool, allowing the detection of high metabolic activity in inflamed plaques [54]. Most interestingly, plaque inflammation is associated with atherothrombotic manifestation, but its relation to serum biomarkers is unclear. In a recently reported post hoc analysis of 130 patients participating in the dal-PLAQUE study, baseline FDG uptake in the most diseased carotid segments positively correlated with blood myeloperoxidase, an effector molecule secreted by granulocytes upon stimulation, in patients suffering from coronary artery disease on statin therapy [55]. In the same cohort, blood levels of IL-6 positively correlated with FDG targetto-background ratio (TBR) in the most diseased carotid segments [56]. Interestingly, hs-CRP, soluble P-selectin and Eselectin, sVICAM1, and MMP-3 and MMP-9 did not correlate with FDG uptake in the carotid artery [55]. Interestingly however in an earlier report by Rudd on 40 patients and colleagues FDG uptake in the carotid artery significantly correlated with serum MMP-9 levels, while a negative correlation was established between FDG TBR in the carotid artery and serum plasminogen activator inhibitor 1 (PAI1) [57]. Again, hs-CRP did not correlate with plaque metabolic activity [57]. The lack of correlation between hs-CRP and plaque inflammation was recently confirmed by immunepathological analysis, that is, the current gold standard, of 160 carotid endarterectomy specimens [58].

Although not strictly considered inflammatory markers due to their principal function as mediators of extracellular matrix remodelling [59], MMPs are gaining a growing interest in the field of atherosclerosis. A recent report suggested that they may become themselves the target of radiolabelled molecular probes for the detection of vulnerable plaques [60]. 


\section{Circulating Lipids, Inflammation, and Plaque Progression}

Plasma low-density lipoprotein cholesterol (LDL-C) level is considered one of the most important risk factors for atherosclerotic plaque instability and several emerging pharmacological approaches are aimed at decreasing vascular inflammation and increasing plaque stability by reducing LDL-C levels [61]. In spite of this, many individuals with evidence of atherosclerotic disease have LDL-C within the normal range, although they might have an altered pattern of lipoproteins subfractions. The concentration of small dense low-density lipoprotein (sdLDL) has been associated with increased cardiovascular risk and with the progression of coronary and carotid atherosclerosis in casecontrol and prospective studies [62-64]. The lipoprotein cholesterol profile and the LDL floatation rate (LDL-RF) were directly and significantly correlated with weight, body mass index, waist, hip, waist/hip ratio, triglycerides, fasting glycaemia, and cIMT and inversely related to high-density lipoprotein cholesterol (HDL-C) [65]. Among the lipoprotein subclasses, triglyceride-rich lipoproteins (TGRL) and sdLDL were shown to independently predict the entity of cIMT and were associated with a proinflammatory activation of peripheral mononuclear cells and endothelial cells [65]. These findings suggest that cholesterol or triglycerides content in lipoproteins could mark specific lipoprotein subclasses with specific atherogenic and proinflammatory properties [66, 67]. The presence of such specific lipoprotein subsets could be also the result of the activation of lipoprotein remodelling enzymes, which occurs during inflammation. For example, the activation of innate immune response results in the reduction of plasma HDL-C levels but also in the remodelling of high-density lipoprotein (HDL) which becomes enriched in proinflammatory mediators and becomes dysfunctional [68-70].

Impaired HDL functionality was also observed in immune-inflammatory and autoimmune diseases $[69,71]$ and was associated with altered lymphocyte subsets distribution and increased cIMT and atherosclerotic plaques [72]. Another key above-mentioned molecule generated during inflammatory processes is the Lp-PLA2, which is associated with increased risk of cardiovascular events [17]. Lp-PLA2 mediates the formation of bioactive mediators (lysophosphatidyl choline and oxidized nonesterified fatty acids) known to elicit several deleterious inflammatory responses involved in the pathobiology of atherosclerosis [73]. Lysophosphatidyl, for example, choline, serves as a potent chemoattractant for monocytes, resulting in foam cell accumulation within the arterial wall [73]. Despite these observations the role of Lp-PLA2, as well as that of the secretory PLA2 (sPLA2), remains enigmatic. In elderly subjects sPLA2 levels, but not Lp-PLA2 levels, were independently associated with atherosclerotic plaques and outcome [74]. Data from the above-mentioned dal-PLAQUE study demonstrated that baseline Lp-PLA2 mass correlated with TBR of FDG uptake in the aorta but not in the carotid artery [55].

A new intriguing molecule related to inflammation and lipid metabolism, the fatty acid binding protein 4 (FABP4 or aP2 in mice), has been identified as a key regulator of core aspects of cardiometabolic disorders, including lipotoxic endoplasmic reticulum stress in macrophages, and macrophage cholesterol trafficking and associated inflammation [75]. In a large study the effects of low-expression variant of FABP 4 were examined at population level $(n=7491)$ and in group of patients with advanced carotid atherosclerosis $(n=92)$ and myocardial infarction $(n=3432)$ [76]. The authors found that the low-expression variant was associated with decreased total cholesterol levels. Furthermore, the carriers of the allelic variant associated with obesity also showed reduced cIMT thickness and lower prevalence of carotid plaques [76]. In another study, plasma levels of FABP4 were also evaluated in relation to symptoms in 59 patients with carotid plaques and 202 having suffered from stroke of another origin [77]. FABP4 levels were higher in patients with carotid atherosclerosis, both systemically and within the atherosclerotic lesion, than in patients having suffered from a stroke from another origin, with particularly high mRNA levels in carotid plaques from patients with the most recent symptoms [77]. Furthermore, FABP4 correlated with the cell-surface markers of monocyte/macrophage lineage CD36, CD68, and CD163 as well as with the presence of CD4positive $\mathrm{T}$ cells in the plaque [78].

Finally, alterations in plasma lipid profile and inflammation are often presenting in deep relation to metabolic status, which could mark the presence of insulin resistance and/or diabetes. While the discussion of the latter is beyond the scope of the present review, it is important to mention that metabolic inflammation could be associated with changes in the circulating profile of key cytokines such as adipokines. To this extent, resistin, leptin, and adiponectin could mark the altered metabolic status which is associated with dyslipidaemia, systemic inflammation, and vascular disease [7983].

\section{Circulating Leukocytes and Plaque Progression}

White blood cells constitute the effector arm of the immune system, attending to both immune surveillance and prompt response to tissue damage. Several cell types are found among white blood cells, each with a different function and differentially activated by specific stimuli. In the last decades, forefront technologies such as polychromatic flow cytometry have allowed the identification of thousands of leukocytes subsets [84], giving the chance to identify alterations in the face of normal white blood cells counts. A careful analysis of circulating leukocytes may thus provide a valuable tool to evaluate the inflammatory and immune status of the patient [85]: using this approach circulating white blood cells may well serve as biomarkers. Among the different cells found to be altered in patients with atherosclerosis, mononuclear cells, both lymphocytes [85] and monocytes [86] subpopulations have been most frequently implicated. In addition, gene expression profiling of leukocytes may provide a valuable tool for the identification of specific types of inflammatory response associated with plaque progression or vulnerability. Interestingly, to date only few studies analyzed the association 
between circulating immune cells and advanced carotid atherosclerotic disease. In fact, most studies concerned with the carotid artery focused on subclinical atherosclerotic disease, such as cIMT. Population-based studies have demonstrated the association between the presence of plaque and total white blood cells $[24,26]$ and monocytes counts $[24,27]$. A recent report on 853 asymptomatic individuals affected by atherosclerosis failed to demonstrate any association between the severity of stenosis or plaque progression at 6 months and white blood cells subtypes [87]. Interestingly, however, neutrophil counts conferred a relative risk of cardiovascular death of 1.9-2.4 in patients bearing a stenosis of $>50 \%$ [87]. Neutrophil count was also shown to strongly associate with the presence of microembolism detected by transcranial Doppler ultrasound in 60 recently symptomatic patients [88].

Monocytes of recently symptomatic patients were shown to bear signs of activations: in particular, they were found to express high concentrations in the adhesion molecules CD11b and thrombospondin 1 [89]. Interestingly, these same markers were shown to correlate with the presence of plateletmonocyte aggregates [89], which appear to be involved in the pathogenesis of atherosclerosis and atherothrombosis $[85,90]$. However, platelet-monocyte aggregates were not shown to associate with the presence of microembolic signals on transcranial Doppler ultrasound in 16 symptomatic and 30 asymptomatic patients affected by carotid atherosclerosis [91]. A subsequent report by Sternberg and colleagues failed to demonstrate an association between higher concentrations of activated monocytes, identified as CD14+CD68+ cells, and the presence of carotid artery atherosclerosis [92]. Notably, patients bearing a carotid plaque had higher activation of $\mathrm{T}$ and $\mathrm{B}$ lymphocytes, identified as CD3+HLA-DR+ and CD20+CD69+, respectively, and higher levels of MMP-9 expression, assessed through mRNA analysis, in peripheral blood mononuclear cells [92]. Consistently, a study by Martin-Ventura et al. showed an increased expression of CD74 (invariant polypeptide of major histocompatibility complex) in peripheral mononuclear blood of patients with carotid artery stenosis [93]. In a recent report on 700 patients selected from the cardiovascular cohort of the Malmö Diet and Cancer study, the percentage of circulating CD19+CD86+ B cells was shown to correlate with higher degree of carotid artery stenosis [94]. In addition, patients with higher percentages of the same B cells subset were prospectively shown to be at higher risk for stroke [94]. Leukocyte telomere length (LTL) is an important determinant of telomere function and cellular replicative capacity, which were both associated with cardiovascular diseases [95, 96]. An association between telomere shortening (TS) and both the progression of atherosclerosis and the incidence of cardiovascular events (CVEs) was recently shown [97]. After adjusting for classical cardiovascular disease risk factors (age, gender, smoking, physical activity, alcohol consumption, systolic blood pressure, glucose levels, lipid profile, and therapies), TS was associated with increased progression of cIMT [97]. Finally, subjects in whom LTL shortened over time showed an increased risk of incident CVE, compared to those in whom LTL lengthened [97]. These data indicate that leukocyte TS is associated with increased risk of subclinical carotid vascular damage and increased incidence of CVEs beyond classical cardiovascular disease risk factors in the general population, whereas LTL lengthening is protective, further connecting leukocytes counts, function, and senescence with atherosclerotic disorders.

\section{Circulating Markers of Inflammation and Intima-Media Thickness}

As mentioned above, thickening of tunica intima and media of the carotid artery, that is, cIMT, is currently considered an early sign of preclinical atherosclerosis. Accordingly, the presence of increased cIMT was shown to increase the risk of future CVEs [98] and to correlate with the presence of traditional cardiovascular risk factors [99]. However, pathogenesis of cIMT is only incompletely understood $[100,101]$ and its relationship with carotid artery plaque is not fully defined. For these reasons, a thorough discussion of the association between inflammatory biomarkers and cIMT does not appear completely relevant to the purpose of the present work. Due to the extreme interest in the subject grown in the last few years, however, we will briefly discuss some of the most interesting results in the field. In the aforementioned study by Chapman et al., plasma levels of IL-6, fibrinogen, and monocytes counts were shown to associate with cIMT [27]. However, the significance of association was lost upon correction for conventional risk factors [27]. In a populationbased sample of 3092 subjects older than 55 years of age, high levels of hs-CRP were associated with higher cIMT [102]. Interestingly, no significant differences in hs-CRP levels were found between patients with subclinical atherosclerosis and patients bearing carotid artery plaques [102]. A recently published meta-analysis comprising 20 studies and 49097 patients showed a significant association between CIMT and serum levels of hs-CRP, fibrinogen, and leukocytes count [22]. Interestingly, MMPs were once again found to associate with vascular disease in the carotid artery: in a report by Orbe and colleague on 400 otherwise healthy subjects, cIMT was shown to positively correlate with serum levels of MMP-10 [103]. When circulating cells are concerned, a recent report on 912 patients from the Multiethnic Study of Atherosclerosis (MESA) demonstrated a higher number of memory $\mathrm{T}$ cells and lower amounts of naïve $\mathrm{T}$ cells in subjects with higher cIMT [104]. Accordingly, higher amounts of CD3+CD4+CD45RA-CD45RO+CCR7- effector memory T cells were found to associate with cIMT [105]. In 622 healthy volunteers CD16+ monocytes were shown to associate both with body mass index (BMI) and with cIMT [106]. The relevance of LTL to cIMT was already discussed above [97].

\section{Markers of Inflammation and Neoangiogenesis}

Progression of atherosclerotic disease with plaque enlargement results in intraplaque hypoxia, which contributes to local inflammation and triggers local neovascularization [107]. The presence of neovessels within atherosclerotic lesions was shown not only to promote plaque growth, but 


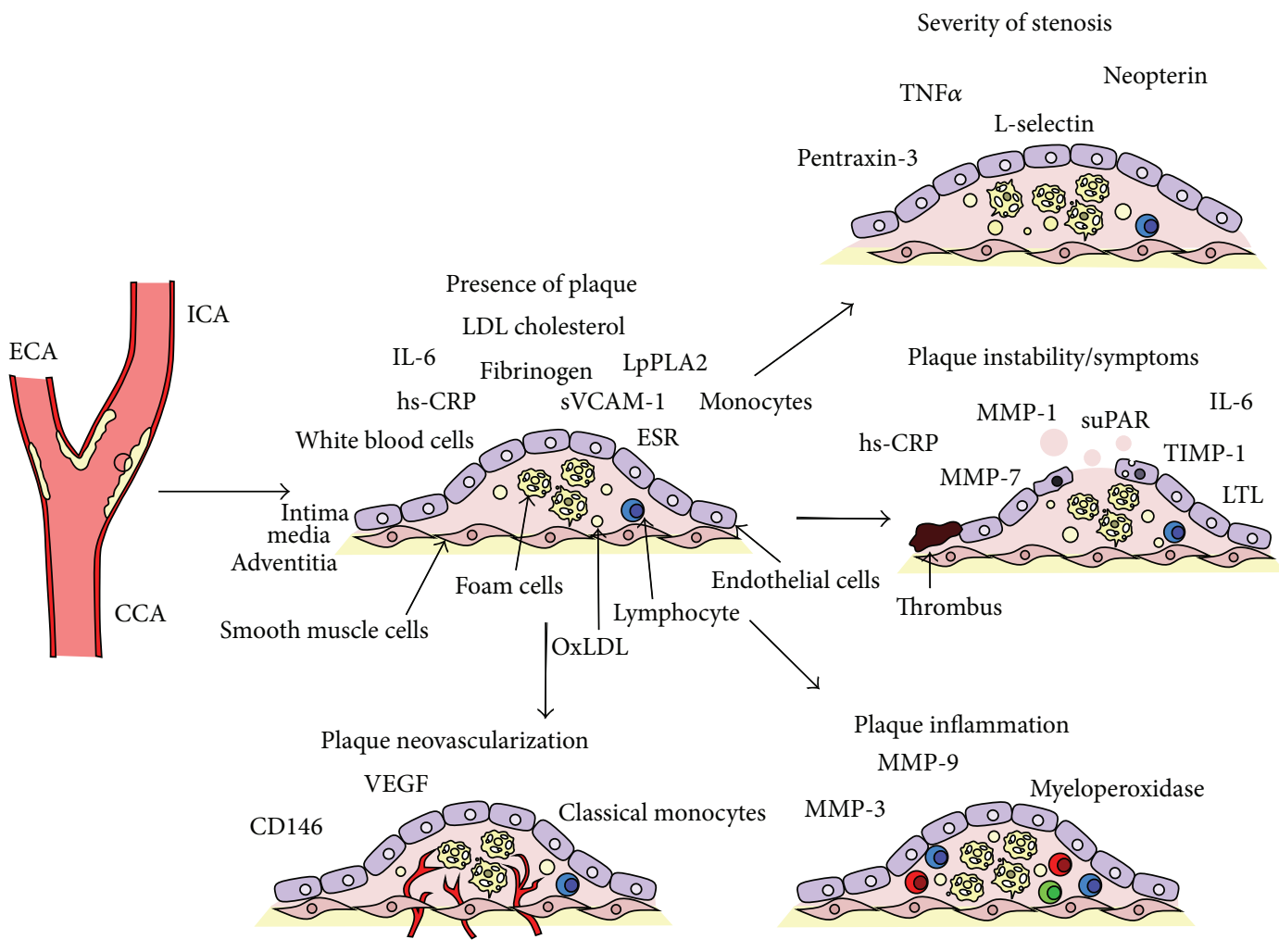

FIGURE 1: Summary of the main biomarkers associated with different stages and manifestation of carotid atherosclerosis. CCA: common carotid artery; ICA: internal carotid artery; ECA: external carotid artery; IL: interleukin; LDL: low-density lipoprotein; OxLDL: oxidized lowdensity lipoprotein; ESR: erythrocyte sedimentation rate; hs-CRP: high sensitivity C-reactive protein; sVCAM-1: soluble vascular cell adhesion molecule 1; LpPLA2: lipoprotein-associated phospholipase A2; TNF- $\alpha$ : tumour necrosis factor alpha; MMP: matrix metalloproteinase; TIMP1: tissue inhibitor of matrix proteinases; suPAR: soluble urokinase plasminogen activator receptor; LTL: leukocyte telomere length; VEGF: vascular endothelial growth factor.

also to contribute to its vulnerability [108]. Thus, in vivo identification of atherosclerotic lesion neovascularization appears to be a suitable approach for the identification of high-risk plaques. Novel noninvasive imaging strategies, in particular contrast-enhanced ultrasound (CEUS), allow the identification of carotid artery plaque neovessels by dynamically assessing plaque uptake of microsized gas-filled particles known as microbubbles $[109,110]$. Currently only few studies assessed the association between the presence of carotid artery plaque neovascularization and circulating biomarkers. A recent report by Qian and colleagues on 40 patients suggests that histologically determined carotid artery vascularization associates with serum levels of CD146 and adhesion molecule of the immunoglobulin superfamily which is implicated in leukocytes extravasation [111]. In another report on 56 patients, the presence of neovessels on carotid plaque surgical specimens positively correlated with serum levels of vascular endothelial growth factor, a master cytokine for angiogenesis and inflammation [112]. Finally, in a recent report Jaipersad and colleagues were able to show a significant correlation between severe plaque neovascularization, detected by CEUS, and circulating levels of classical CD14++CD16-CCR2+ monocytes [113]. In addition, circulating monocytes were shown to express high levels of proangiogenic molecules, such as Tie2 [113].

\section{Markers of Inflammation and Therapeutic Intervention}

To date, several population-based preventive programs aimed at cardiovascular risk reduction were able to substantially abate cardiovascular morbidity and mortality [114, 115]. Most importantly, the introduction of statin therapy was able to reduce cardiovascular mortality by over one-third [116]. Interestingly, aside from statin therapy main effect on cholesterol lowering, statins were shown to reduce the concentration of circulating markers of inflammation [117]. However, a substantial risk needs to be addressed and novel therapeutic targets are needed, alongside novel therapeutic endpoints to assess its efficacy. Assessing intraplaque inflammation and its response to treatment may provide a short term secondary endpoint for clinical studies. In a study by Tang and colleagues, for example, the institution of high dose statin therapy was associated with lowering of carotid artery plaque inflammation assessed through magnetic resonance imaging (MRI) after six and twelve weeks of follow-up [118]. A report by Corti et al. confirms these results and interestingly shows an association between the entity of LDL cholesterol reduction and the disappearance of wall features of inflammation in MRI [119]. In the recently published dalPLAQUE study, the FDG TBR in the most diseased carotid 


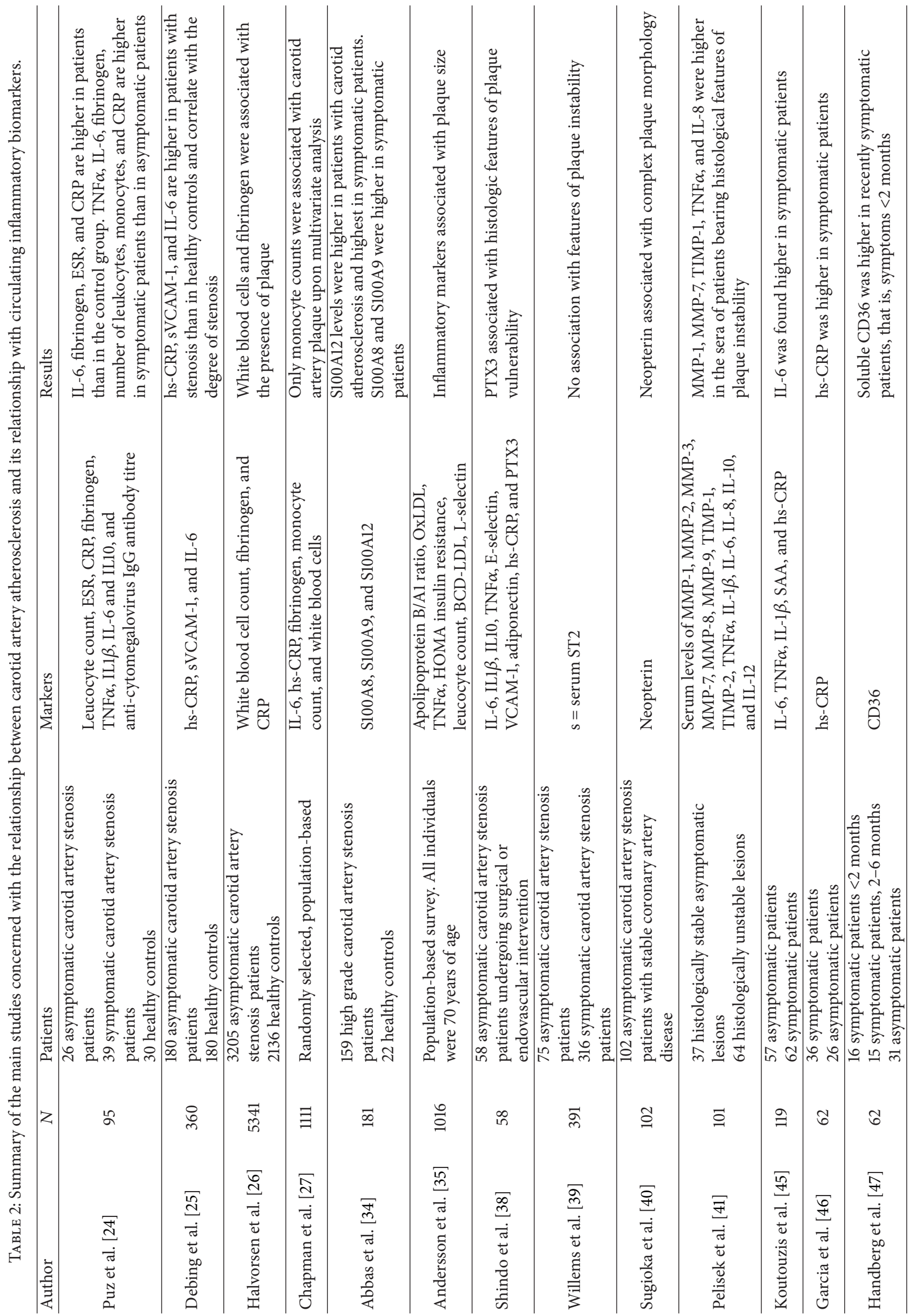




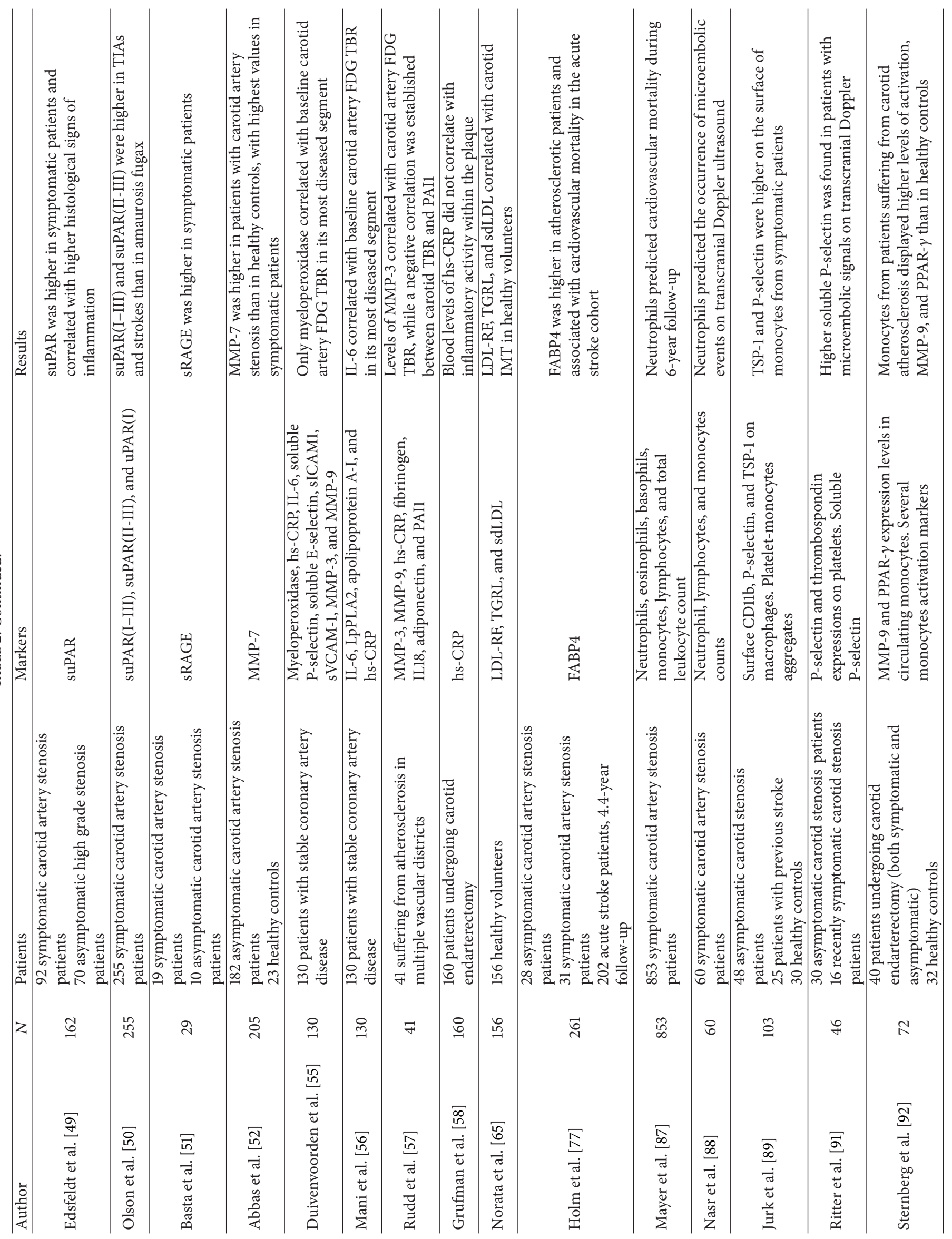




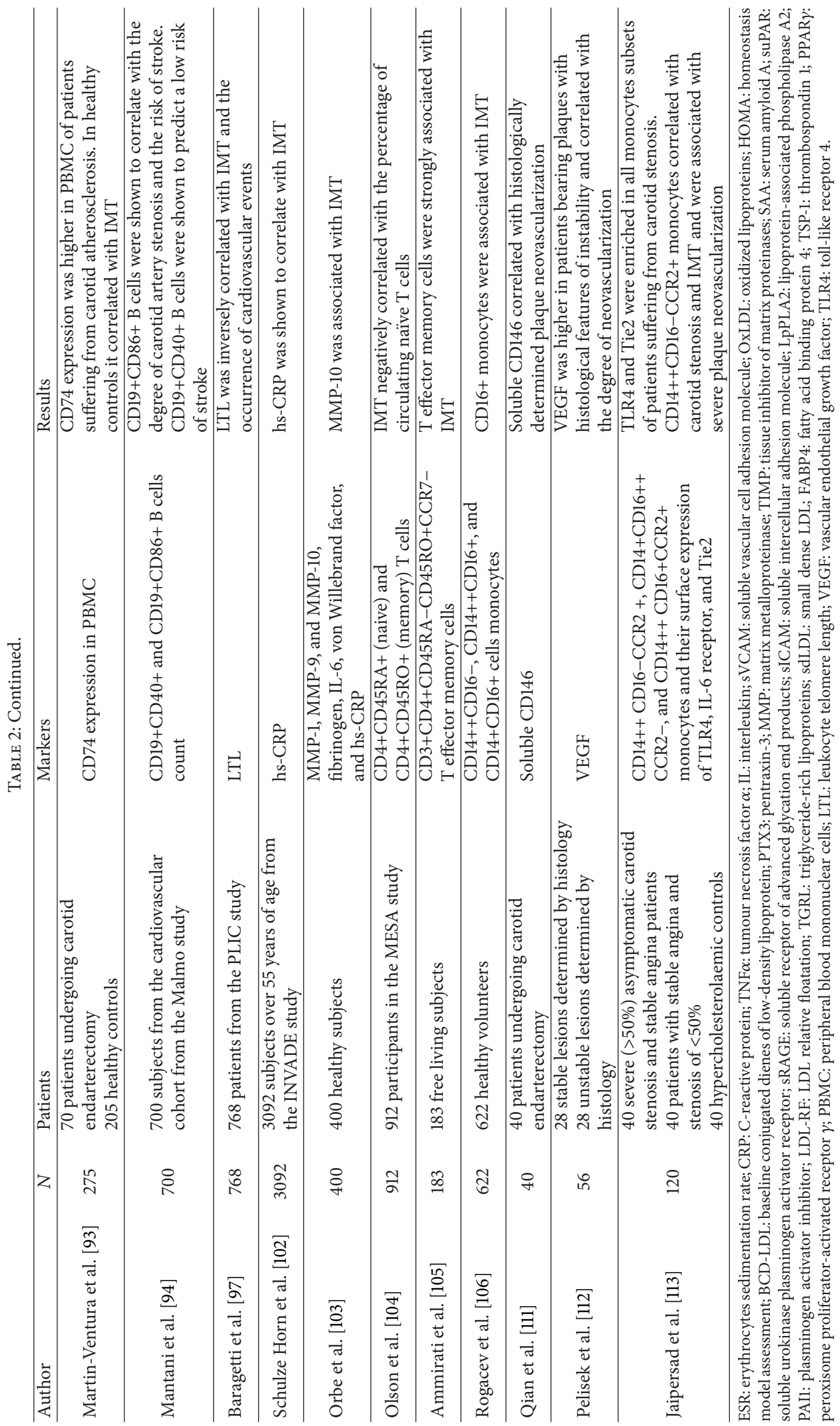


artery segment was shown to be significantly reduced after 6 months of Dalcetrapib, which modulates cholesteryl ester transfer protein activity to raise high-density lipoprotein cholesterol [120].

\section{Conclusions}

Carotid atherosclerosis is highly prevalent in the general population and exerts a high toll in terms of disability and mortality. Currently, the therapeutic management of carotid plaques strongly relies on the severity of stenosis as the primary guide for choosing the appropriate intervention. However, this approach has proved largely unsatisfactory [11]. Inflammation is a basic pathogenic element in the development of atherosclerotic disease and in its manifestation [53]. The detection of the inflammatory and immune profile of the patient may lead to a better stratification of asymptomatic patients, but also of patients at risk for stroke recurrence [121], beyond the simple evaluation of stenosis. In particular, master cytokines such as IL- 6 and $\mathrm{TNF} \alpha$ were shown to reliably predict the presence and the characteristics of the plaque, while novel markers such as the members of the MMP family appear to be implicated in its destabilization. Inflammatory biomarkers may also provide a tool to longitudinally follow patients in order to assess their response to therapy, allowing a better selection of appropriate strategy on the path towards personalized medicine. Table 2 provides a summary of the studies concerned with the association between inflammatory biomarkers and carotid atherosclerosis while Figure 1 graphically recapitulates the relation between biomarkers and carotid plaque evolution and complications.

\section{Conflict of Interests}

The authors declare that the research was conducted in the absence of any commercial or financial relationships that could be construed as a potential conflict of interests.

\section{Authors' Contribution}

Enrico Ammirati, Francesco Moroni, and Giuseppe Danilo Norata wrote the report. Marco Magnoni and Paolo G. Camici critically revised the paper.

\section{Acknowledgment}

Enrico Ammirati received financial support from the "Giovane Ricercatore 2009 Grant" from Italian Health Ministry (Project code GR-2009-1608780).

\section{References}

[1] M. de Weerd, J. P. Greving, B. Hedblad et al., "Prevalence of asymptomatic carotid artery stenosis in the general population: an individual participant data meta-analysis," Stroke, vol. 41, no. 6, pp. 1294-1297, 2010.

[2] A. G. den Hartog, S. Achterberg, F. L. Moll et al., "Asymptomatic carotid artery stenosis and the risk of ischemic stroke according to subtype in patients with clinical manifest arterial disease," Stroke, vol. 44, no. 4, pp. 1002-1007, 2013.
[3] J. W. Norris, C. Z. Zhu, N. M. Bornstein, and B. R. Chambers, "Vascular risks of asymptomatic carotid stenosis," Stroke, vol. 22, no. 12, pp. 1485-1490, 1991.

[4] D. Inzitari, M. Eliasziw, P. Gates et al., "The causes and risk of stroke in patients with asymptomatic internal-carotid-artery stenosis. North American Symptomatic Carotid Endarterectomy Trial Collaborators," The New England Journal of Medicine, vol. 342, no. 23, pp. 1693-1700, 2000.

[5] G. W. Petty, R. D. Brown Jr., J. P. Whisnant, J. D. Sicks, W. M. O'Fallon, and D. O. Wiebers, "Ischemic stroke subtypes: a population-based study of incidence and risk factors," Stroke, vol. 30, no. 12, pp. 2513-2516, 1999.

[6] P. M. Rothwell, R. Gibson, and C. P. Warlow, "Interrelation between plaque surface morphology and degree of stenosis on carotid angiograms and the risk of ischemic stroke in patients with symptomatic carotid stenosis," Stroke, vol. 31, no. 3, pp. 615$621,2000$.

[7] M. Tendera, V. Aboyans, M. Bartelink et al., "ESC Guidelines on the diagnosis and treatment of peripheral artery diseases: document covering atherosclerotic disease of extracranial carotid and vertebral, mesenteric, renal, upper and lower extremity arteries: the Task Force on the Diagnosis and Treatment of Peripheral Artery Diseases of the European Society of Cardiology (ESC)," European Heart Journal, vol. 32, no. 22, pp. 28512906, 2011.

[8] O. Benavente, D. Moher, and B. Pham, "Carotid endarterectomy for asymptomatic carotid stenosis: a meta-analysis," British Medical Journal, vol. 317, no. 7171, pp. 1477-1480, 1998.

[9] G. Raman, D. Moorthy, N. Hadar et al., "Management strategies for asymptomatic carotid stenosis: a systematic review and meta-analysis," Annals of Internal Medicine, vol. 158, no. 9, pp. 676-685, 2013.

[10] J. D. Spence, "Asymptomatic carotid stenosis," Circulation, vol. 127, no. 6, pp. 739-742, 2013.

[11] E. Ammirati, M. Magnoni, and P. G. Camici, "Need for new non-invasive imaging strategies to identify high-risk asymptomatic patients with carotid stenosis," International Journal of Cardiology, vol. 168, no. 4, pp. 4342-4343, 2013.

[12] S. C. Johnston, E. S. O’Meara, T. A. Manolio et al., "Cognitive impairment and decline are associated with carotid artery disease in patients without clinically evident cerebrovascular disease," Annals of Internal Medicine, vol. 140, no. 4, pp. 237-247, 2004.

[13] L. Buratti, C. Balucani, G. Viticchi et al., "Cognitive deterioration in bilateral asymptomatic severe carotid stenosis," Stroke, vol. 45, no. 7, pp. 2072-2077, 2014.

[14] S. Balestrini, C. Perozzi, C. Altamura et al., "Severe carotid stenosis and impaired cerebral hemodynamics can influence cognitive deterioration," Neurology, vol. 80 , no. 23, pp. 21452150, 2013.

[15] G. Jayasooriya, A. Thapar, J. Shalhoub, and A. H. Davies, "Silent cerebral events in asymptomatic carotid stenosis," Journal of Vascular Surgery, vol. 54, no. 1, pp. 227-236, 2011.

[16] H. H. Oei, I. M. van der Meer, A. Hofman et al., "Lipoproteinassociated phospholipase A2 activity is associated with risk of coronary heart disease and ischemic stroke: the Rotterdam Study," Circulation, vol. 111, no. 5, pp. 570-575, 2005.

[17] V. Nambi, R. C. Hoogeveen, L. Chambless et al., "Lipoproteinassociated phospholipase $\mathrm{A}_{2}$ and high-sensitivity C-reactive protein improve the stratification of ischemic stroke risk in the atherosclerosis risk in communities (ARIC) study," Stroke, vol. 40, no. 2, pp. 376-381, 2009. 
[18] P. M. Ridker, M. Cushman, M. J. Stampfer et al., "Inflammation, aspirin, and the risk of cardiovascular disease in apparently healthy men," The New England Journal of Medicine, vol. 336, no. 14, pp. 973-979, 1997.

[19] P. M. Ridker, J. E. Buring, J. Shih, M. Matias, and C. H. Hennekens, "Prospective study of C-reactive protein and the risk of future cardiovascular events among apparently healthy women," Circulation, vol. 98, no. 8, pp. 731-733, 1998.

[20] N. S. Rost, P. A. Wolf, C. S. Kase et al., "Plasma concentration of C-reactive protein and risk of ischemic stroke and transient ischemic attack: the Framingham Study," Stroke, vol. 32, no. 11, pp. 2575-2579, 2001.

[21] M. W. Lorenz, J. F. Polak, M. Kavousi et al., "Carotid intimamedia thickness progression to predict cardiovascular events in the general population (the PROG-IMT collaborative project): a meta-analysis of individual participant data," The Lancet, vol. 379, no. 9831, pp. 2053-2062, 2012.

[22] P. Willeit, S. G. Thompson, S. Agewall et al., "Inflammatory markers and extent and progression of early atherosclerosis: meta-analysis of individual-participant-data from 20 prospective studies of the PROG-IMT Collaboration," European Journal of Preventive Cardiology, 2014.

[23] L. Hermus, J. D. Lefrandt, R. A. Tio, J.-C. Breek, and C. J. Zeebregts, "Carotid plaque formation and serum biomarkers," Atherosclerosis, vol. 213, no. 1, pp. 21-29, 2010.

[24] P. Puz, A. Lasek-Bal, D. Ziaja, Z. Kazibutowska, and K. Ziaja, "Inflammatory markers in patients with internal carotid artery stenosis," Archives of Medical Science, vol. 9, no. 2, pp. 254-260, 2013.

[25] E. Debing, E. Peeters, C. Demanet, M. de Waele, and P. van den Brande, "Markers of inflammation in patients with symptomatic and asymptomatic carotid artery stenosis: a casecontrol study," Vascular and Endovascular Surgery, vol. 42, no. 2, pp. 122-127, 2008.

[26] D. S. Halvorsen, S. H. Johnsen, E. B. Mathiesen, and I. Njølstad, "The association between inflammatory markers and carotid atherosclerosis is sex dependent: the Tromsø study," Cerebrovascular Diseases, vol. 27, no. 4, pp. 392-397, 2009.

[27] C. M. L. Chapman, J. P. Beilby, B. M. McQuillan, P. L. Thompson, and J. Hung, "Monocyte count, but not C-reactive protein or interleukin-6, is an independent risk marker for subclinical carotid atherosclerosis," Stroke, vol. 35, no. 7, pp. 1619-1624, 2004.

[28] G. D. Norata, C. Garlanda, and A. L. Catapano, "The long pentraxin PTX3: a modulator of the immunoinflammatory response in atherosclerosis and cardiovascular diseases," Trends in Cardiovascular Medicine, vol. 20, no. 2, pp. 35-40, 2010.

[29] F. Bonacina, A. Baragetti, A. L. Catapano, and G. D. Norata, "Long pentraxin 3: experimental and clinical relevance in cardiovascular diseases," Mediators of Inflammation, vol. 2013, Article ID 725102, 10 pages, 2013.

[30] G. D. Norata, P. Marchesi, A. Pirillo et al., "Long pentraxin 3, a key component of innate immunity, is modulated by high-density lipoproteins in endothelial cells," Arteriosclerosis, Thrombosis, and Vascular Biology, vol. 28, no. 5, pp. 925-931, 2008.

[31] G. D. Norata, P. Marchesi, V. K. Pulakazhi Venu et al., "Deficiency of the long pentraxin PTX3 promotes vascular inflammation and atherosclerosis," Circulation, vol. 120, no. 8, pp. 699-708, 2009.

[32] M. Knoflach, S. Kiechl, A. Mantovani et al., "Pentraxin-3 as a marker of advanced atherosclerosis results from the Bruneck,
ARMY and ARFY Studies," PLoS ONE, vol. 7, no. 2, Article ID e31474, 2012.

[33] A. Baragetti, M. Knoflach, I. Cuccovillo et al., "Pentraxin 3 (PTX3) plasma levels and carotid intima media thickness progression in the general population," Nutrition, Metabolism and Cardiovascular Diseases, vol. 24, no. 5, pp. 518-523, 2014.

[34] A. Abbas, P. Aukrust, T. B. Dahl et al., "High levels of S100A12 are associated with recent plaque symptomatology in patients with carotid atherosclerosis," Stroke, vol. 43, no. 5, pp. 1347-1353, 2012.

[35] J. Andersson, J. Sundström, L. Kurland et al., "The carotid artery plaque size and echogenicity are related to different cardiovascular risk factors in the elderly: the prospective investigation of the vasculature in uppsala seniors (PIVUS) study," Lipids, vol. 44, no. 5, pp. 397-403, 2009.

[36] P. G. Camici, O. E. Rimoldi, O. Gaemperli, and P. Libby, "Non-invasive anatomic and functional imaging of vascular inflammation and unstable plaque," European Heart Journal, vol. 33, no. 11, pp. 1309-1317, 2012.

[37] A. V. Finn, M. Nakano, J. Narula, F. D. Kolodgie, and R. Virmani, "Concept of vulnerable/unstable plaque," Arteriosclerosis, Thrombosis, and Vascular Biology, vol. 30, no. 7, pp. 1282-1292, 2010.

[38] A. Shindo, H. Tanemura, K. Yata et al., "Inflammatory biomarkers in atherosclerosis: pentraxin 3 can become a novel marker of plaque vulnerability," PLOS ONE, vol. 9, no. 6, Article ID e100045, 2014.

[39] S. Willems, P. H. A. Quax, G. J. de Borst et al., "Soluble ST2 levels are not associated with secondary cardiovascular events and vulnerable plaque phenotype in patients with carotid artery stenosis," Atherosclerosis, vol. 231, no. 1, pp. 48-53, 2013.

[40] K. Sugioka, T. Naruko, T. Hozumi et al., "Elevated levels of neopterin are associated with carotid plaques with complex morphology in patients with stable angina pectoris," Atherosclerosis, vol. 208, no. 2, pp. 524-530, 2010.

[41] J. Pelisek, M. Rudelius, P. Zepper et al., "Multiple biological predictors for vulnerable carotid lesions," Cerebrovascular Diseases, vol. 28, no. 6, pp. 601-610, 2009.

[42] Z. S. Galis and J. J. Khatri, "Matrix metalloproteinases in vascular remodeling and atherogenesis: the good, the bad, and the ugly," Circulation Research, vol. 90, no. 3, pp. 251-262, 2002.

[43] J. L. Johnson, N. P. Jenkins, W. C. Huang et al., "Relationship of MMP-14 and TIMP-3 expression with macrophage activation and human atherosclerotic plaque vulnerability," Mediators of Inflammation, vol. 2014, Article ID 276457, 17 pages, 2014.

[44] C. Cheng, D. Tempel, R. van Haperen et al., "Activation of MMP8 and MMP13 by angiotensin II correlates to severe intraplaque hemorrhages and collagen breakdown in atherosclerotic lesions with a vulnerable phenotype," Atherosclerosis, vol. 204, no. 1, pp. 26-33, 2009.

[45] M. Koutouzis, L. S. Rallidis, G. Peros et al., "Serum interleukin6 is elevated in symptomatic carotid bifurcation disease," Acta Neurologica Scandinavica, vol. 119, no. 2, pp. 119-125, 2009.

[46] B. A. Garcia, C. Ruiz, P. Chacon, J. A. Sabin, and M. Matas, "High-sensitivity C-reactive protein in high-grade carotid stenosis: risk marker for unstable carotid plaque," Journal of Vascular Surgery, vol. 38, no. 5, pp. 1018-1024, 2003.

[47] A. Handberg, M. Skjelland, A. E. Michelsen et al., "Soluble CD36 in plasma is increased in patients with symptomatic atherosclerotic carotid plaques and is related to plaque instability," Stroke, vol. 39, no. 11, pp. 3092-3095, 2008. 
[48] D. C. Rijken and H. R. Lijnen, "New insights into the molecular mechanisms of the fibrinolytic system," Journal of Thrombosis and Haemostasis, vol. 7, no. 1, pp. 4-13, 2009.

[49] A. Edsfeldt, M. Nitulescu, H. Grufman et al., "Soluble urokinase plasminogen activator receptor is associated with inflammation in the vulnerable human atherosclerotic plaque," Stroke, vol. 43, no. 12, pp. 3305-3312, 2012.

[50] F. J. Olson, T. Thurison, M. Ryndel, G. Høyer-Hansen, and B. Fagerberg, "Soluble urokinase-type plasminogen activator receptor forms in plasma as markers of atherosclerotic plaque vulnerability," Clinical Biochemistry, vol. 43, no. 1-2, pp. 124-130, 2010.

[51] G. Basta, M. Castagnini, S. del Turco et al., "High plasma levels of the soluble receptor for advanced glycation endproducts in patients with symptomatic carotid atherosclerosis," European Journal of Clinical Investigation, vol. 39, no. 12, pp. 1065-1072, 2009.

[52] A. Abbas, P. Aukrust, D. Russell et al., "Matrix metalloproteinase 7 is associated with symptomatic lesions and adverse events in patients with carotid atherosclerosis," PLoS ONE, vol. 9, no. 1, Article ID e84935, 2014.

[53] P. Libby, "Inflammation in atherosclerosis," Arteriosclerosis, Thrombosis, and Vascular Biology, vol. 32, no. 9, pp. 2045-2051, 2012.

[54] E. Ammirati, F. Moroni, P. Pedrotti et al., "Non-invasive imaging of vascular inflammation," Frontiers in Immunology, vol. 5, article 399, 2014.

[55] R. Duivenvoorden, V. Mani, M. Woodward et al., "Relationship of serum inflammatory biomarkers with plaque inflammation assessed by FDG PET/CT: the dal-PLAQUE study," JACC: Cardiovascular Imaging, vol. 6, no. 10, pp. 1087-1094, 2013.

[56] V. Mani, M. Woodward, D. Samber et al., "Predictors of change in carotid atherosclerotic plaque inflammation and burden as measured by 18-FDG-PET and MRI, respectively, in the dalPLAQUE study," The International Journal of Cardiovascular Imaging, vol. 30, no. 3, pp. 571-582, 2014.

[57] J. H. F. Rudd, K. S. Myers, S. Bansilal et al., "Relationships among regional arterial inflammation, calcification, risk factors, and biomarkers: a prospective fluorodeoxyglucose positronemission tomography/computed tomography imaging study," Circulation: Cardiovascular Imaging, vol. 2, no. 2, pp. 107-115, 2009.

[58] H. Grufman, I. Gonçalves, A. Edsfeldt et al., "Plasma levels of high-sensitive C-reactive protein do not correlate with inflammatory activity in carotid atherosclerotic plaques," Journal of Internal Medicine, vol. 275, no. 2, pp. 127-133, 2014.

[59] C. Bonnans, J. Chou, and Z. Werb, "Remodelling the extracellular matrix in development and disease," Nature Reviews Molecular Cell Biology, vol. 15, no. 12, pp. 786-801, 2014.

[60] A. Müller, S. D. Krämer, R. Meletta et al., "Gene expression levels of matrix metalloproteinases in human atherosclerotic plaques and evaluation of radiolabeled inhibitors as imaging agents for plaque vulnerability," Nuclear Medicine and Biology, vol. 41, no. 7, pp. 562-569, 2014.

[61] G. D. Norata, C. M. Ballantyne, and A. L. Catapano, "New therapeutic principles in dyslipidaemia: focus on LDL and Lp(a) lowering drugs," European Heart Journal, vol. 34, no. 24, pp. 1783-1789, 2013.

[62] M. A. Austin, J. L. Breslow, C. H. Hennekens, J. E. Buring, W. C. Willett, and R. M. Krauss, "Low-density lipoprotein subclass patterns and risk of myocardial infarction," The Journal of the American Medical Association, vol. 260, no. 13, pp. 1917-1921, 1988.
[63] M. J. Stampfer, R. M. Krauss, J. Ma et al., "A prospective study of triglyceride level, low-density lipoprotein particle diameter, and risk of myocardial infarction," Journal of the American Medical Association, vol. 276, no. 11, pp. 882-888, 1996.

[64] A. C. St-Pierre, B. Cantin, G. R. Dagenais et al., "Low-density lipoprotein subfractions and the long-term risk of ischemic heart disease in men: 13-year follow-up data from the Québec Cardiovascular Study," Arteriosclerosis, Thrombosis, and Vascular Biology, vol. 25, no. 3, pp. 553-559, 2005.

[65] G. D. Norata, S. Raselli, L. Grigore et al., "Small dense LDL and VLDL predict common carotid artery IMT and elicit an inflammatory response in peripheral blood mononuclear and endothelial cells," Atherosclerosis, vol. 206, no. 2, pp. 556-562, 2009.

[66] S. Koba, T. Hirano, T. Kondo et al., "Significance of small dense low-density lipoproteins and other risk factors in patients with various types of coronary heart disease," American Heart Journal, vol. 144, no. 6, pp. 1026-1035, 2002.

[67] G. D. Norata, L. Grigore, S. Raselli et al., "Post-prandial endothelial dysfunction in hypertriglyceridemic subjects: molecular mechanisms and gene expression studies," Atherosclerosis, vol. 193, no. 2, pp. 321-327, 2007.

[68] A. L. Catapano, A. Pirillo, F. Bonacina, and G. D. Norata, "HDL in innate and adaptive immunity," Cardiovascular Research, vol. 103, no. 3, pp. 372-383, 2014.

[69] G. D. Norata, A. Pirillo, E. Ammirati, and A. L. Catapano, "Emerging role of high density lipoproteins as a player in the immune system," Atherosclerosis, vol. 220, no. 1, pp. 11-21, 2012.

[70] A. Pirillo, A. L. Catapano, and G. D. Norata, "HDL in infectious diseases and sepsis," in High Density Lipoproteins, vol. 224 of Handbook of Experimental Pharmacology, pp. 483-508, Springer, Berlin, Germany, 2015.

[71] F. Montecucco, E. Favari, G. D. Norata, N. Ronda, J.-R. Nofer, and N. Vuilleumier, "Impact of systemic inflammation and autoimmune diseases on apoA-I and HDL plasma levels and functions," in High Density Lipoproteins, vol. 224 of Handbook of Experimental Pharmacology, pp. 455-482, 2015.

[72] E. Ammirati, E. P. Bozzolo, R. Contri et al., "Cardiometabolic and immune factors associated with increased common carotid artery intima-media thickness and cardiovascular disease in patients with systemic lupus erythematosus," Nutrition, Metabolism and Cardiovascular Diseases, vol. 24, no. 7, pp. 751759, 2014.

[73] C. K. Glass and J. L. Witztum, "Atherosclerosis: the road ahead," Cell, vol. 104, no. 4, pp. 503-516, 2001.

[74] L. Lind, T. Simon, L. Johansson et al., "Circulating levels of secretory- and lipoprotein-associated phospholipase $\mathrm{A}_{2}$ activities: relation to atherosclerotic plaques and future all-cause mortality," European Heart Journal, vol. 33, no. 23, pp. 29462954, 2012.

[75] J. M. Ordovas, "Identification of a functional polymorphism at the adipose fatty acid binding protein gene (FABP4) and demonstration of its association with cardiovascular disease: a path to follow," Nutrition Reviews, vol. 65, no. 3, pp. 130-134, 2007.

[76] J. Saksi, P. Ijas, M. I. Mayranpaa et al., "Low-expression variant of fatty acid-binding protein 4 favors reduced manifestations of atherosclerotic disease and increased plaque stability," Circulation: Cardiovascular Genetics, vol. 7, no. 5, pp. 588-598, 2014.

[77] S. Holm, T. Ueland, T. B. Dahl et al., "Fatty Acid binding protein 4 is associated with carotid atherosclerosis and outcome in patients with acute ischemic stroke," PLoS ONE, vol. 6, no. 12, Article ID e28785, 2011. 
[78] H. E. Agardh, L. Folkersen, J. Ekstrand et al., "Expression of fatty acid-binding protein $4 / \mathrm{aP} 2$ is correlated with plaque instability in carotid atherosclerosis," Journal of Internal Medicine, vol. 269, no. 2, pp. 200-210, 2011.

[79] G. D. Norata, I. Baragetti, S. Raselli et al., "Plasma adiponectin levels in chronic kidney disease patients: relation with molecular inflammatory profile and metabolic status," Nutrition, Metabolism and Cardiovascular Diseases, vol. 20, no. 1, pp. 5663, 2010.

[80] G. D. Norata, S. Raselli, L. Grigore et al., "Leptin: adiponectin ratio is an independent predictor of intima media thickness of the common carotid artery," Stroke, vol. 38, no. 10, pp. 28442846, 2007.

[81] G. D. Norata, M. Ongari, K. Garlaschelli, S. Raselli, L. Grigore, and A. L. Catapano, "Plasma resistin levels correlate with determinants of the metabolic syndrome," European Journal of Endocrinology, vol. 156, no. 2, pp. 279-284, 2007.

[82] F. Molica, S. Morel, B. R. Kwak, F. Rohner-Jeanrenaud, and S. Steffens, "Adipokines at the crossroad between obesity and cardiovascular disease," Thrombosis and Haemostasis, vol. 113, no. 3, pp. 553-566, 2015.

[83] J. Van de Voorde, B. Pauwels, C. Boydens, and K. Decaluwé, "Adipocytokines in relation to cardiovascular disease," Metabolism: Clinical and Experimental, vol. 62, no. 11, pp. 1513-1521, 2013.

[84] S. P. Perfetto, P. K. Chattopadhyay, and M. Roederer, "Seventeen-colour flow cytometry: unravelling the immune system," Nature Reviews Immunology, vol. 4, no. 8, pp. 648-655, 2004.

[85] E. Ammirati, F. Moroni, M. Magnoni, and P. G. Camici, "The role of $\mathrm{T}$ and $\mathrm{B}$ cells in human atherosclerosis and atherothrombosis," Clinical \& Experimental Immunology, vol. 179, no. 2, pp. 173-187, 2015.

[86] A. S. Jaipersad, G. Y. H. Lip, S. Silverman, and E. Shantsila, “The role of monocytes in angiogenesis and atherosclerosis," Journal of the American College of Cardiology, vol. 63, no. 1, pp. 1-11, 2014.

[87] F. J. Mayer, D. Gruenberger, M. Schillinger et al., "Prognostic value of neutrophils in patients with asymptomatic carotid artery disease," Atherosclerosis, vol. 231, no. 2, pp. 274-280, 2013.

[88] N. Nasr, J. B. Ruidavets, J. F. Arnal, P. Sie, and V. Larrue, "Association of neutrophil count with microembolization in patients with symptomatic carotid artery stenosis," Atherosclerosis, vol. 207, no. 2, pp. 519-523, 2009.

[89] K. Jurk, M. A. Ritter, C. Schriek et al., "Activated monocytes capture platelets for heterotypic association in patients with severe carotid artery stenosis," Thrombosis and Haemostasis, vol. 103, no. 6, pp. 1193-1202, 2010.

[90] S. Massberg, K. Brand, S. Grüner et al., "A critical role of platelet adhesion in the initiation of atherosclerotic lesion formation," The Journal of Experimental Medicine, vol. 196, no. 7, pp. 887896, 2002.

[91] M. A. Ritter, K. Jurk, C. Schriek et al., "Microembolic signals on transcranial doppler ultrasound are correlated with platelet activation markers, but not with platelet-leukocyte associates: a study in patients with acute stroke and in patients with asymptomatic carotid stenosis," Neurological Research, vol. 31, no. 1, pp. 11-16, 2009.

[92] Z. Sternberg, H. Ghanim, K. M. Gillotti et al., "Flow cytometry and gene expression profiling of immune cells of the carotid plaque and peripheral blood," Atherosclerosis, vol. 229, no. 2, pp. 338-347, 2013.
[93] J. L. Martin-Ventura, J. Madrigal-Matute, B. Munoz-Garcia et al., "Increased CD74 expression in human atherosclerotic plaques: contribution to inflammatory responses in vascular cells," Cardiovascular Research, vol. 83, no. 3, pp. 586-594, 2009.

[94] P. T. Mantani, I. Ljungcrantz, L. Andersson et al., "Circulating $\mathrm{CD} 0^{+}$and $\mathrm{CD} 6^{+} \mathrm{B}$ cell subsets demonstrate opposing associations with risk of stroke," Arteriosclerosis, Thrombosis, and Vascular Biology, vol. 34, no. 1, pp. 211-218, 2014.

[95] S. W. Brouilette, J. S. Moore, A. D. McMahon et al., "Telomere length, risk of coronary heart disease, and statin treatment in the West of Scotland Primary Prevention Study: a nested casecontrol study," The Lancet, vol. 369, no. 9556, pp. 107-114, 2007.

[96] S. Brouilette, R. K. Singh, J. R. Thompson et al., "White cell telomere length and risk of premature myocardial infarction," Arteriosclerosis, Thrombosis, and Vascular Biology, vol. 23, no. 5, pp. 842-846, 2003.

[97] A. Baragetti, J. Palmen, K. Garlaschelli et al., “Telomere shortening over 6 years is associated with increased subclinical carotid vascular damage and worse cardiovascular prognosis in the general population," Journal of Internal Medicine, vol. 277, no. 4, pp. 478-487, 2015.

[98] H. M. den Ruijter, S. A. Peters, T. J. Anderson et al., "Common carotid intima-media thickness measurements in cardiovascular risk prediction: a meta-analysis," The Journal of the American Medical Association, vol. 308, no. 8, pp. 796-803, 2012.

[99] P. H. Davis, J. D. Dawson, W. A. Riley, and R. M. Lauer, "Carotid intimal-medial thickness is related to cardiovascular risk factors measured from childhood through middle age the muscatine Study," Circulation, vol. 104, no. 23, pp. 2815-2819, 2001.

[100] V. A. Korshunov and B. C. Berk, "Strain-dependent vascular remodeling: the 'Glagov phenomenon' is genetically determined," Circulation, vol. 110, no. 2, pp. 220-226, 2004.

[101] E. M. Smolock, V. A. Korshunov, G. Glazko, X. Qiu, J. Gerloff, and B. C. Berk, "Ribosomal protein L17, RpL17, is an inhibitor of vascular smooth muscle growth and carotid intima formation," Circulation, vol. 126, no. 20, pp. 2418-2427, 2012.

[102] C. Schulze Horn, R. Ilg, K. Sander et al., "High-sensitivity Creactive protein at different stages of atherosclerosis: results of the INVADE study," Journal of Neurology, vol. 256, no. 5, pp. 783-791, 2009.

[103] J. Orbe, I. Montero, J. A. Rodríguez, O. Beloqui, C. Roncal, and J. A. Páramo, "Independent association of matrix metalloproteinase-10, cardiovascular risk factors and subclinical atherosclerosis," Journal of Thrombosis and Haemostasis, vol. 5, no. 1, pp. 91-97, 2007.

[104] N. C. Olson, M. F. Doyle, N. S. Jenny et al., "Decreased naive and increased memory $\mathrm{CD} 4^{+} \mathrm{T}$ cells are associated with subclinical atherosclerosis: the multi-ethnic study of atherosclerosis," PLoS ONE, vol. 8, no. 8, Article ID e71498, 2013.

[105] E. Ammirati, D. Cianflone, V. Vecchio et al., "Effector memory T cells are associated with atherosclerosis in humans and animal models," Journal of the American Heart Association, vol. 1, no. 111, pp. 27-41, 2012.

[106] K. S. Rogacev, C. Ulrich, L. Blömer et al., "Monocyte heterogeneity in obesity and subclinical atherosclerosis," European Heart Journal, vol. 31, no. 3, pp. 369-376, 2010.

[107] H. M. Kwon, G. Sangiorgi, E. L. Ritman et al., "Enhanced coronary vasa vasorum neovascularization in experimental hypercholesterolemia," The Journal of Clinical Investigation, vol. 101, no. 8, pp. 1551-1556, 1998.

[108] R. Virmani, F. D. Kolodgie, A. P. Burke et al., "Atherosclerotic plaque progression and vulnerability to rupture: angiogenesis 
as a source of intraplaque hemorrhage," Arteriosclerosis, Thrombosis, and Vascular Biology, vol. 25, no. 10, pp. 2054-2061, 2005.

[109] S. Coli, M. Magnoni, G. Sangiorgi et al., "Contrast-enhanced ultrasound imaging of intraplaque neovascularization in carotid arteries: correlation with histology and plaque echogenicity," Journal of the American College of Cardiology, vol. 52, no. 3, pp. 223-230, 2008.

[110] M. Magnoni, S. Coli, M. M. Marrocco-Trischitta et al., "Contrast-enhanced ultrasound imaging of periadventitial vasa vasorum in human carotid arteries," European Journal of Echocardiography, vol. 10, no. 2, pp. 260-264, 2009.

[111] Y.-N. Qian, Y.-T. Luo, H.-X. Duan et al., "Adhesion molecule CD146 and its soluble form correlate well with carotid atherosclerosis and plaque instability," CNS Neuroscience \& Therapeutics, vol. 20, no. 5, pp. 438-445, 2014.

[112] J. Pelisek, G. Well, C. Reeps et al., "Neovascularization and angiogenic factors in advanced human carotid artery stenosis," Circulation Journal, vol. 76, no. 5, pp. 1274-1282, 2012.

[113] A. S. Jaipersad, A. Shantsila, G. Y. Lip, and E. Shantsila, "Expression of monocyte subsets and angiogenic markers in relation to carotid plaque neovascularization in patients with pre-existing coronary artery disease and carotid stenosis," Annals of Medicine, vol. 46, no. 7, pp. 530-538, 2014.

[114] F. Turnbull, "Effects of different blood-pressure-lowering regimens on major cardiovascular events: results of prospectivelydesigned overviews of randomised trials," The Lancet, vol. 362, no. 9395, pp. 1527-1535, 2003.

[115] C. M. Fichtenberg and S. A. Glantz, "Association of the California tobacco control program with declines in cigarette consumption and mortality from heart disease," The New England Journal of Medicine, vol. 343, no. 24, pp. 1772-1777, 2000.

[116] B. Mihaylova, J. Emberson, L. Blackwell et al., "The effects of lowering LDL cholesterol with statin therapy in people at low risk of vascular disease: meta-analysis of individual data from 27 randomised trials," The Lancet, vol. 380, no. 9841, pp. 581590, 2012.

[117] P. M. Ridker, E. Danielson, F. A. Fonseca et al., "Reduction in Creactive protein and LDL cholesterol and cardiovascular event rates after initiation of rosuvastatin: a prospective study of the JUPITER trial," The Lancet, vol. 373, no. 9670, pp. 1175-1182, 2009.

[118] T. Y. Tang, S. P. S. Howarth, S. R. Miller et al., "The ATHEROMA (Atorvastatin Therapy: Effects on Reduction of Macrophage Activity) Study. Evaluation using ultrasmall superparamagnetic iron oxide-enhanced magnetic resonance imaging in carotid disease," Journal of the American College of Cardiology, vol. 53, no. 22, pp. 2039-2050, 2009.

[119] R. Corti, V. Fuster, Z. A. Fayad et al., "Effects of aggressive versus conventional lipid-lowering therapy by simvastatin on human atherosclerotic lesions: a prospective, randomized, doubleblind trial with high-resolution magnetic resonance imaging," Journal of the American College of Cardiology, vol. 46, no. 1, pp. 106-112, 2005.

[120] Z. A. Fayad, V. Mani, M. Woodward et al., "Safety and efficacy of dalcetrapib on atherosclerotic disease using novel non-invasive multimodality imaging (dal-PLAQUE): a randomised clinical trial," The Lancet, vol. 378, no. 9802, pp. 1547-1559, 2011.

[121] M. Marnane, S. Prendeville, C. McDonnell et al., "Plaque inflammation and unstable morphology are associated with early stroke recurrence in symptomatic carotid stenosis," Stroke, vol. 45, no. 3, pp. 801-806, 2014. 


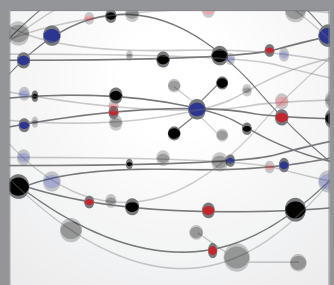

The Scientific World Journal
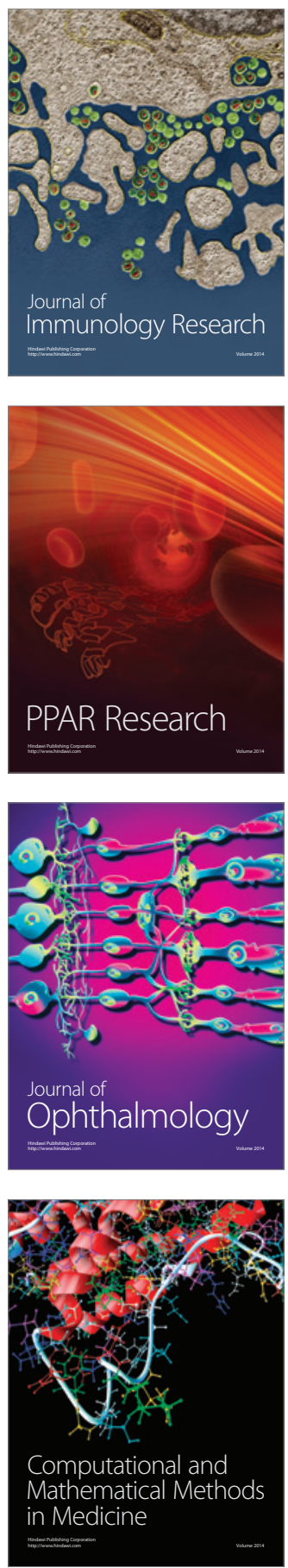

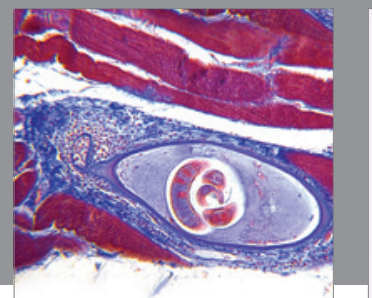

Gastroenterology

Research and Practice
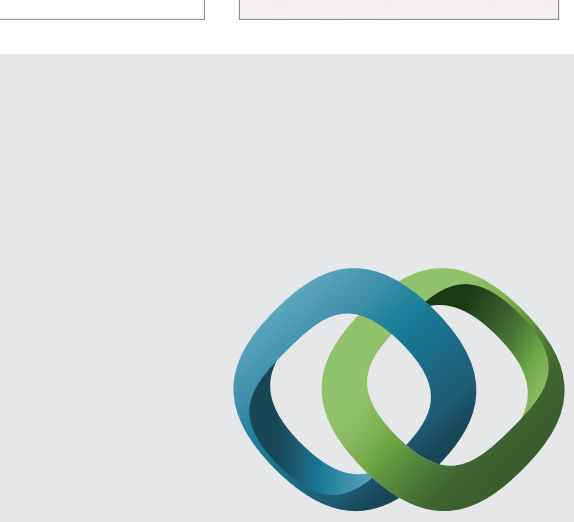

\section{Hindawi}

Submit your manuscripts at

http://www.hindawi.com
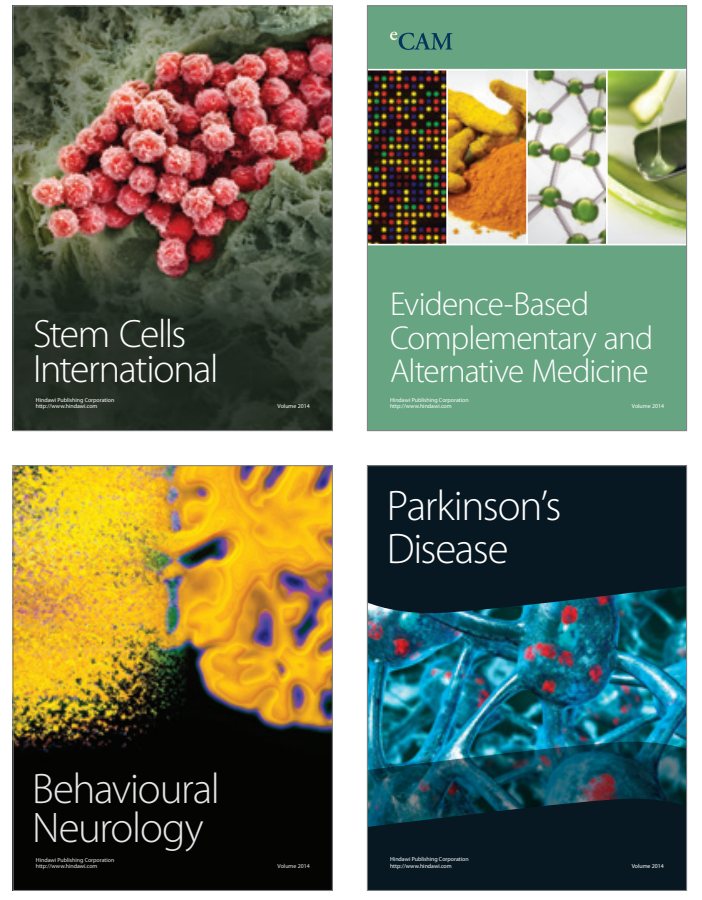
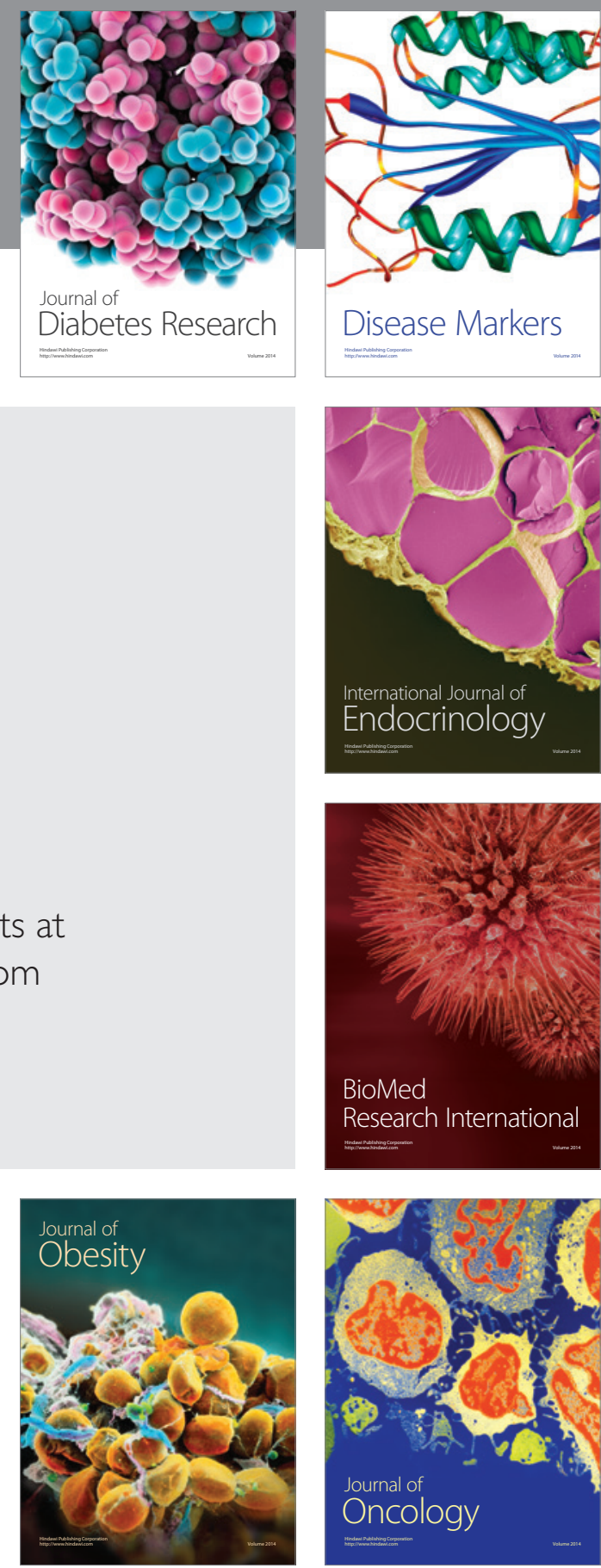

Disease Markers
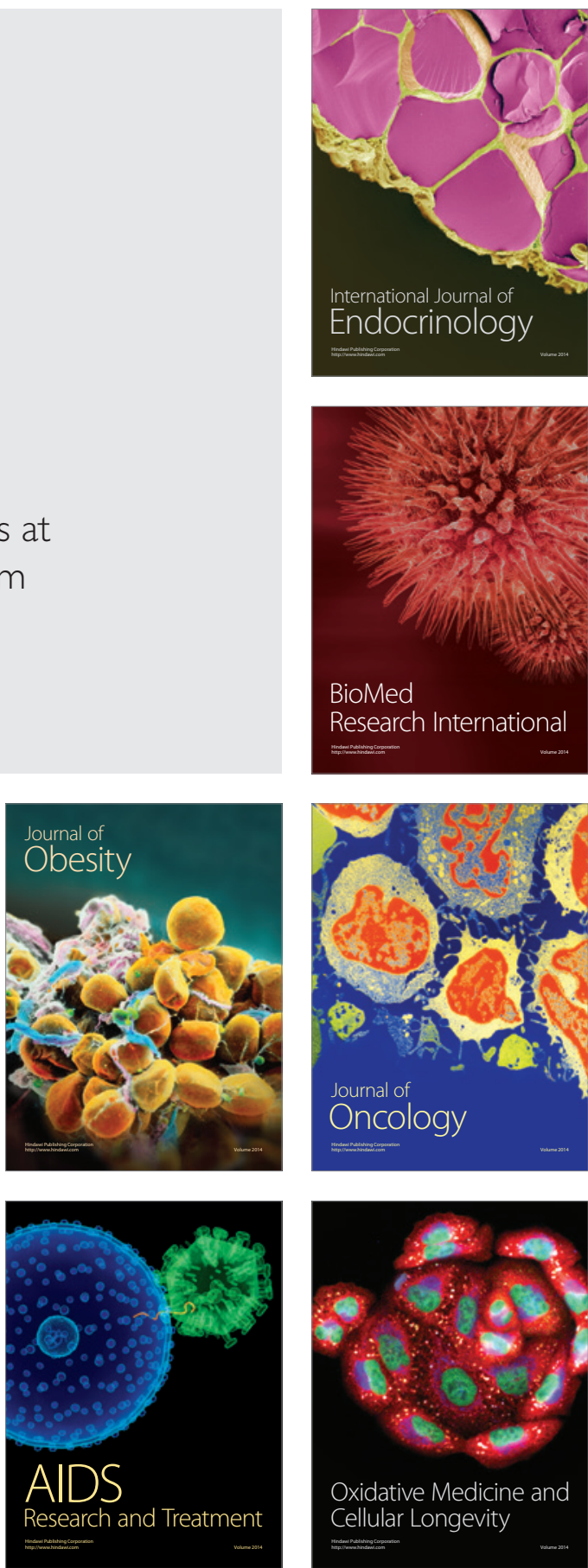